Variable High-Order Multiblock

Overlapping Grid Methods for Mixed Steady and Unsteady Multiscale Viscous Flows, Part II: Hypersonic Nonequilibrium Flows

A. Lani, B. Sjogreen, H. C. Yee, W. D. Henshaw

July 1,2011

42nd AIAA Plasmadynamics and Lasers Conference Honolulu, HI, United States

June 27, 2011 through June 30, 2011 
This document was prepared as an account of work sponsored by an agency of the United States government. Neither the United States government nor Lawrence Livermore National Security, LLC, nor any of their employees makes any warranty, expressed or implied, or assumes any legal liability or responsibility for the accuracy, completeness, or usefulness of any information, apparatus, product, or process disclosed, or represents that its use would not infringe privately owned rights. Reference herein to any specific commercial product, process, or service by trade name, trademark, manufacturer, or otherwise does not necessarily constitute or imply its endorsement, recommendation, or favoring by the United States government or Lawrence Livermore National Security, LLC. The views and opinions of authors expressed herein do not necessarily state or reflect those of the United States government or Lawrence Livermore National Security, LLC, and shall not be used for advertising or product endorsement purposes. 


\title{
Variable high-order multiblock overlapping grid methods for mixed steady and unsteady multiscale viscous flows, part II: hypersonic nonequilibrium flows
}

\author{
Andrea Lani, ${ }^{*}$ Björn Sjögreen ${ }^{\dagger}$ H.C. Yee ${ }^{\ddagger}$ and William D. Henshaw ${ }^{\S}$
}

\begin{abstract}
The variable high-order multiblock overlapping (overset) grids method of Sjögreen \& Yee (CiCP, Vol.5, 2008) for a perfect gas has been extended to nonequilibrium flows. This work makes use of the recently developed high-order well-balanced shock-capturing schemes and their filter counterparts (Wang et al., J. Comput. Phys., 2009, 2010) that exactly preserve certain non-trivial steady state solutions of the chemical nonequilibrium governing equations. Multiscale turbulence with strong shocks and flows containing both steady and unsteady components is best treated by mixing of numerical methods and switching on the appropriate scheme in the appropriate subdomains of the flow fields, even under the multiblock grid or adaptive grid refinement framework. While low dissipative sixth- or higher-order shock-capturing filter methods are appropriate for unsteady turbulence with shocklets, second- and third-order shock-capturing methods are more effective for strong steady or nearly steady shocks in terms of convergence. It is anticipated that our variable high-order overset grid framework capability with its highly modular design will allow an optimum synthesis of these new algorithms in such a way that the most appropriate spatial discretizations can be tailored for each particular region of the flow. In this paper some of the latest developments in single block high-order filter schemes for chemical nonequilibrium flows are applied to overset grid geometries. The numerical approach is validated on a number of test cases characterized by hypersonic conditions with strong shocks, including the reentry flow surrounding a 3D Apollo-like NASA Crew Exploration Vehicle that might contain mixed steady and unsteady components, depending on the flow conditions.
\end{abstract}

\section{Motivation, objectives and validation process}

The time-accurate unsteady 3D compressible flow solver ADPDIS3D is supported by a grant from the Department of Energy (DOE) SciDAC program through the Science Application Partnership (SAP) initiative. The objective of this grant is to develop, implement and validate this variable high-order 3D multiblock overlapping (overset) grid solver for problems involving turbulence with strong shocks and density variations. ADPDIS3D includes capabilities for both Direct Numerical Simulation (DNS), resolving all scales of the flow fields, and Large Eddy Simulation (LES), modeling the small turbulent scales. One of the unique features of the solver is its ability to perform DNS and LES computations in non-trivial geometries through the use of overset curvilinear grids. ADPDIS3D contains a large number of high-order finite difference schemes and shock-capturing schemes. These schemes can be used to perform accurate unsteady computations for flow speeds that range from nearly incompressible to hypersonic speeds. ${ }^{1-6}$ Importantly, the code implements many innovative low dissipative algorithms that adaptively use numerical dissipation from shock-capturing schemes as postprocessing filters on non-dissipative high-order centered schemes. ${ }^{1-6}$ These filter schemes were especially designed for improved accuracy over standard high-order shock-capturing schemes in capturing turbulence with strong shocks and density variations. For multi-dimensional curvilinear grids, the metrics are evaluated at the same high-order as the spatial base scheme with high-order freestream preservation. ${ }^{7}$

*Postdoctoral Fellow, Stanford Centre for Turbulence Research / NASA Ames; alani0@stanford.edu

${ }^{\dagger}$ Computational Scientist, Lawrence Livermore National Lab; sjogreen2@llnl.gov

${ }^{\ddagger}$ Senior Research Scientist, MS 258-5, NASA Ames Research Center; helen.m.yee@nasa.gov

$\S$ Computational Scientist, Lawrence Livermore National Lab; henshaw@llnl.gov 
Recently, these filter schemes were proved to be well-balanced schemes ${ }^{6}$ in the sense that these schemes exactly preserve certain non-trivial steady-state solutions of the chemical nonequilibrium governing equations. With this added property the filter schemes can better minimize spurious numerics in reacting flows containing both steady shocks and unsteady turbulence with shocklet components than standard non-wellbalanced shock-capturing schemes. For a description of the algorithms and their performance, including results of a detailed LES computation of temporal-evolving mixing layers, see e.g. ${ }^{1-6,8}$ Furthermore, ADPDIS3D contains three choices of solvers: standard compressible flow, compressible non-ideal MHD ${ }^{2}$ and chemical nonequilibrium hypersonic flows. ${ }^{6}$ For a more detailed description see. ${ }^{1,9}$ In order to improve numerical stability, a preprocessing step by conditioning the governing equations via entropy splitting, ${ }^{10}$ Ducros et al. splitting ${ }^{11}$ or system form of the Tadmor entropy conserving form ${ }^{12}$ is included in the procedure.

Multiscale turbulence with strong shocks and flows containing both steady and unsteady components require mixing of numerical methods and switching on the appropriate scheme in the appropriate subdomains of the flow fields, even under the multiblock grid or adaptive grid refinement framework. It is a non-trivial task to find adaptive schemes that both correctly identify different flow features and switching locally to a suitable method without seriously affecting the overall accuracy of the method. While low dissipative sixth- or higher-order shock-capturing filter methods are appropriate for unsteady turbulence with shocklets, third-order or lower shock-capturing methods are more effective for strong steady or nearly steady shocks in terms of convergence. An important application for the subject flow physics is chemical and thermo-chemical nonequilibrium hypersonic turbulence flows. In order to minimize the shortcomings of low order and high-order shock-capturing schemes for the subject flows, ADPDIS3D can utilize overset grids with different types of spatial schemes and orders of accuracy on different chosen grid blocks as an efficient method in combating the difficulty. It is anticipated that this particular overset grid framework capability with its highly modular design will allow an optimum synthesis of these new algorithms in such a way that the most appropriate spatial discretizations can be tailored for each particular region of the flow. In addition, frameworks have been built and are operational in ADPDIS3D to interface with (1) the MUTATION library (version 1.3, Thierry Magin, private communication) for more accurate transport, and chemical and thermodynamics properties for nonequilibrium flows than standard table look up and mixture rules, and (2) the overset grid generator Ogen ${ }^{13}$ that is part of the Overture platform. ${ }^{14}$ Ogen can be used to generate overlapping grids for high-order accurate approximations that use wide stencils and require high-order accurate interpolation.

\section{A. Objectives}

The overset grid implementation for a perfect gas has been validated in Sjögreen \& Yee. ${ }^{1}$ The objective of the current investigation is a follow-up on previous work by Sjögreen \& Yee ${ }^{1}$ to validate the overset grid implementation for nonequilibrium flows that were initiated in the work by Wang, Yee, Sjögreen \& $S^{6} u^{6}$ for a single block grid implementation. With additional improvements ${ }^{6}$ the same numerical methods and the same overset grid framework for perfect gases can be carried over to chemical and certain thermo-chemical nonequilibrium flows. Inviscid 1D flows with relatively weak shocks and 2D smooth flows on a single block grid were considered in. ${ }^{6,15}$ Here the investigation is extended to both inviscid and viscous flows with strong shocks on 2D and 3D multiblock overlapping grids. A 5-species and one-temperature air model in chemical nonequilibrium is considered in all cases.

Second-order TVD, fifth- or higher-order WENO schemes are applied on and around the bow shock in combination with fourth-order or sixth-order filter schemes elsewhere. ${ }^{2,3}$ A 2-D blunt body is considered for the present validation of both inviscid and viscous flows. In this test case, the flow consists of a major steady bow shock and smooth flow on the rest of the computational domain. Unlike the standard pseudo timemarching to the steady state, in order to assess the capability of unsteady computations, the computations are time accurate even though the chosen test cases are laminar.

Some preliminary results of a mixed steady/unsteady nonequilibrium 3D computation with variable order numerical schemes on an Apollo-like Crew Exploration Vehicle (CEV) are included for the solver ADPDIS3D code validation purposes. This is a work in progress of the first several stages of a multistage validation process for the nonequilibrium implementation. The following gives a brief discussion of the complexity in the validation process and indicates the status of the current investigation relative to our overarching goal. 


\section{B. Validation process}

In order to validate the solver for realistic physics, the entire validation process is rather involved. A brief outline on the validation process consists of the following stages:

\section{2D inviscid nonequilibrium flows}

- Single grid vs. overset grid

- Overset grid using the same scheme on all blocks vs. different schemes on different blocks

\section{Viscous nonequilibrium flow counterparts of I}

\section{3D validation of I and II}

IV. More realistic test cases for turbulence with strong shocks for I-III

The current paper addresses I and II with some work in progress for III.

\section{Flow Solver}

\section{A. Governing equations}

The system of governing equations for a gas mixture in thermodynamic equilibrium and chemical nonequilibrium can be expressed in conservative form as:

$$
\mathbf{U}_{t}+\left(\mathbf{F}_{k}(\mathbf{U})\right)_{x_{k}}+\left(\mathbf{G}_{k}(\mathbf{U})\right)_{x_{k}}=\mathbf{S}(\mathbf{U}), \quad k=1, . ., 3,
$$

where $\mathbf{U}=\left(\rho_{s}, \rho \mathbf{v}, \rho E\right)^{T}$ are the conservative variables, and $\rho_{s}$ the partial densities with $s=1, \ldots, N_{s}$ for a mixture of $N_{s}$ species. The convective and diffusive fluxes, $\mathbf{F}_{k}$ and $\mathbf{G}_{k}$ are

$$
\mathbf{F}_{\mathbf{k}}=\left(\begin{array}{c}
\rho_{s} v_{k} \\
\rho v_{k} v_{l}+p \delta_{k l} \\
\rho v_{k} H
\end{array}\right), \quad \mathbf{G}_{\mathbf{k}}=\left(\begin{array}{c}
\rho_{s} v_{s k} \\
-\tau_{k l} \\
-\tau_{k l} v_{l}+q_{k}+\sum_{s} \rho_{s} v_{s k} h_{s}
\end{array}\right), \quad l=1, . ., 3 .
$$

where $h_{s}$ and $H$ are the species and total enthalpy per unity mass, $v$ are the velocity components and $v_{s k}$ are the diffusion velocities. The mixture total density, the pressure and the total energy per unit volume appearing in (2) are

$$
\rho=\sum_{s} \rho_{s}, \quad p=R T \sum_{s=1}^{N_{s}} \frac{\rho_{s}}{M_{s}}, \quad \rho E=\sum_{s=1}^{N_{s}} \rho_{s}\left(e_{s}(T)+h_{s}^{0}\right)+\frac{1}{2} \rho v^{2},
$$

where $R$ is the universal gas constant, $h_{s}^{0}$ are the species formation enthalpies, and $M_{s}$ indicates the species molar masses. The exact expressions for the species internal energies $e_{s}(T)$, including roto-translational, vibrational and electronic contributions, can be found in. ${ }^{16}$ The viscous stresses $\tau_{k l}$ and the conduction heat fluxes $q_{k}$ can be expressed as follows:

$$
\tau_{k l}=\mu\left[\left(\frac{\partial u_{l}}{\partial x_{k}}+\frac{\partial u_{k}}{\partial x_{l}}\right)-\frac{2}{3} \nabla \cdot \mathbf{v} \delta_{k l}\right], \quad q_{k}=-\left(\lambda^{t}+\lambda^{r}\right) \frac{\partial T}{\partial x_{k}} .
$$

The computation of transport coefficients and fluxes is based on the modified Chapman-Enskog perturbative analysis for partially ionized plasmas and on efficient iterative algorithms ${ }^{17}$ for solving the linear systems from which shear viscosity $\mu$ and translational thermal conductivity $\lambda^{t}$ can be obtained. The rotational thermal conductivity $\lambda^{r}$ is calculated with the Eucken approximation. ${ }^{18}$ The diffusion fluxes $\rho_{s} v_{s k}$ are computed by solving the Stefan-Maxwell system, ${ }^{16}$ i.e. a linear system in the diffusion fluxes consisting of as many equations as the mixture chemical species, supplemented by the auxiliary condition of zero sum for the diffusion fluxes. The source term vector in (1) is

$$
\mathbf{S}=\left(\begin{array}{c}
\dot{\omega}_{s} \\
0 \\
0
\end{array}\right), \quad \dot{\omega}_{s}=M_{s} \sum_{r=1}^{N r}\left(\nu_{s r}^{\prime \prime}-\nu_{s r}^{\prime}\right)\left\{k_{f r} \prod_{m=1}^{N s}\left(\frac{\rho_{m}}{M_{m}}\right)^{\nu_{m r}^{\prime}}-k_{b r} \prod_{m=1}^{N s}\left(\frac{\rho_{m}}{M_{m}}\right)^{\nu_{m r}^{\prime \prime}}\right\}
$$


with $\dot{\omega}_{s}$ expressing the mass production/destruction term for chemical species $s$. In (5), $\nu$ are the stoichiometric coefficients and the forward reaction rates coefficients $k_{f r}=A_{f, r} T^{n_{f, r}} \exp \left(-E_{f, r} / k T\right)$ are expressed by means of Arrhenius' law. The backward reactions rates coefficients are computed as $k_{b, r}=k_{f, r} / K_{c, r}^{e q}$, where $K_{c, r}^{e q}$ is the equilibrium constant. In the present work, all thermodynamic, transport and chemical properties have been calculated by the MUTATION library (version 1.3, Thierry Magin) which provides state-of-the-art models described extensively in ${ }^{16}$ and. ${ }^{17}$ Interested readers are referred to these references for details.

\section{B. Finite difference discretizations}

In spite of the large number of low dissipative high-order schemes contained in ADPDIS3D that have been extensively validated for a perfect gas and for several $1 \mathrm{D}$ and $2 \mathrm{D}$ nonequilibrium flow test cases ${ }^{1-6}$ the present study only considers Harten-Yee TVD ${ }^{19}$ fifth and seventh-order WENO-Lax Friedrichs (WENO5-LF, WENO7-LF) ${ }^{6,15}$ and fourth-order or sixth-order filter central finite difference schemes ${ }^{2,3}$ for the numerical experiments to illustrate the concept. In particular, WENO-LF schemes have been used on single block grids or in blocks enclosing the bow shock to discretize the convective fluxes. On overset grid blocks not enclosing the shock, the dissipative portion of WENO5 has been utilized as a high-order nonlinear filter for sixth-order central base schemes (WENO5fi). In viscous computations, matching order of central discretization as the convection flux derivatives is used for the viscous flux derivatives. In all simulations a pointwise evaluation of the source term has been applied. The explicit second- or fourth-order Runge-Kutta method is used in a time-accurate mode for the time discretization. Due to the explicit time-accurate computation, a very large number of iterations should be expected to reach steady state. Since time accuracy is not a concern for the 2-D blunt body flow as it consists of a major bow shock and smooth flow on the remainder of the flow field, a second-order Runge-Kutta temporal discretization (RK2) is employed for the 2D test case, except for the variable order cases which have been run with the classical fourth-order Runge-Kutta method (RK4). When high-order spatial schemes are used for the 3D CEV computations, RK4 would be more appropriate. With a sufficiently fine grid, unsteady features of the CEV flow field, if they exist, can be observed with this time-accurate approach.

\section{Finite difference WENO schemes}

Consider a scalar hyperbolic conservation law with a source term in one dimension:

$$
u_{t}+f(u)_{x}=s(u) .
$$

with a positive advection speed $\partial f(u) / \partial u \geq 0$. For a finite difference discretization, the point values $u_{i}$ at mesh points $x_{i}$ are evolve in time with a uniform mesh size $\Delta x$ for simplicity. The spatial derivative in (6) is approximated by a conservative flux difference:

$$
\left.f(u)_{x}\right|_{x=x_{i}} \approx \frac{1}{\Delta x}\left(\tilde{f}_{i+\frac{1}{2}}-\tilde{f}_{i-\frac{1}{2}}\right)
$$

The numerical flux $\tilde{f}_{i+\frac{1}{2}}$ is computed through the neighboring point values $f_{j}=f\left(u_{j}\right)$. For a $(2 k-1)$-th order WENO scheme, first $k$ numerical fluxes are computed by

$$
\tilde{f}_{i+\frac{1}{2}}^{(r)}=\sum_{j=0}^{k-1} c_{r j} f\left(x_{i-r+j}\right), \quad r=0, \ldots, k-1
$$

corresponding to $k$ different candidate stencils $S_{r}(i)=x_{i-r}, \ldots, x_{i-r+k-1}$. Each of these $k$ numerical fluxes is $k$-th order accurate. For example, when $k=3$ (fifth-order WENO (WENO5)), the three third order accurate numerical fluxes are given by

$$
\begin{aligned}
\tilde{f}_{i+\frac{1}{2}}^{(0)} & =\frac{1}{3} f_{i}+\frac{5}{6} f_{i+1}-\frac{1}{6} f_{i+2} \\
\tilde{f}_{i+\frac{1}{2}}^{(1)} & =-\frac{1}{6} f_{i-1}+\frac{5}{6} f_{i}+\frac{1}{3} f_{i+1} \\
\tilde{f}_{i+\frac{1}{2}}^{(2)} & =\frac{1}{3} f_{i-2}-\frac{7}{6} f_{i-1}+\frac{11}{6} f_{i} .
\end{aligned}
$$

\section{4 of 16}

American Institute of Aeronautics and Astronautics 
The $(2 k-1)$-th order WENO flux consists of a convex combination of all these $k$ fluxes:

$$
\tilde{f}_{i+\frac{1}{2}}=\sum_{r=0}^{k-1} w_{r} \tilde{f}_{i+\frac{1}{2}}^{(r)}
$$

The conditions $w_{r} \geq 0$ and $\sum_{r=0}^{k-1} w_{r}=1$ hold for the nonlinear weights which are defined as

$$
w_{r}=\frac{\alpha_{r}}{\sum_{s=0}^{k-1} \alpha_{s}}, \quad \alpha_{r}=\frac{d_{r}}{\left(\epsilon+\beta_{r}\right)^{2}} .
$$

The linear weights, $d_{r}$, yield the required $(2 k-1)$-th order accuracy, and $\beta_{r}$ are the smoothness indicators of the stencil $S_{r}(i)$ which measure the smoothness of the function $f(u(x))$ within the stencil. $\epsilon$ is a constant which prevents the denominator from becoming zero (typically assumed to be $10^{-6}$ ). An example of linear weights and smoothness indicators for $k=3$ can be found in. ${ }^{20}$ The procedure for the case with $f^{\prime}(u) \leq 0$ is mirror symmetric with respect to $i+\frac{1}{2}$. An upwinding mechanism, necessary for stabilizing the scheme, can be embodied in the WENO schemes by a global flux splitting, such as Roe with entropy fix (WENO-RF) or Lax-Friedrichs, as described in. ${ }^{20}$ The latter, in particular, is defined as

$$
f^{ \pm}(u)=\frac{1}{2}(f(u) \pm \lambda \alpha u), \quad \alpha=\max _{u}\left|f^{\prime}(u)\right|, \quad \lambda=1 .
$$

The WENO procedure is applied to both $f^{+}$and $f^{-}$with upwind biased stencils. The scheme for which the max is taken globally along the line of computation is commonly denoted WENO-LF. A well balanced version (WENO-LF-WB) of the baseline WENO-LF, capable of preserving certain non-trivial steady-state solutions exactly and resolving accurately relatively small perturbations around the steady solution is introduced in. ${ }^{15}$ In one form of WENO-LF-WB, in order not to affect stability near shocks, $\lambda$ is no longer constant but acts as an equilibrium limiter:

$$
\lambda:=\max \left(\min \left(1, \chi_{1}\right), \ldots, \min \left(1, \chi_{m}\right)\right), \quad \chi_{j}=\frac{\left(\left|r_{j}^{+}\right|+\left|r_{j}^{-}\right|\right)^{2}}{\left|r_{j}^{+}\right|^{2}+\left|r_{j}^{-}\right|^{2}+\epsilon},
$$

with $r_{j}^{ \pm}=r_{j}\left(u_{i \pm 1}, x_{i}\right)-r_{j}\left(u_{i}, x_{i}\right)$ being differences of some known functions (e.g. computed pressure or density) which should be close to zero near the specific steady state. $\epsilon$ is again a small quantity $\left(10^{-6}\right)$ to avoid division by zero. Both baseline WENO-LF (fifth-, seventh-, ninth-order) and WENO-LF-WB (fifthand seventh- order) schemes have been employed for our numerical experiments. More details can be found in. ${ }^{6,15}$

\section{Well-Balanced High-Order Filter Schemes for Reacting Flows ${ }^{1-6}$}

Part of the inaccuracy in direct numerical simulations (DNS) and large eddy simulations (LES) of turbulent flow using standard high-order shock-capturing schemes is due to the fact that this type of computation involves long time integrations. Standard stability and accuracy theories in numerical analysis are not applicable to long time wave propagations and/or long time integrations. ${ }^{21}$ The original construction of modern shock-capturing schemes were developed for rapidly developing unsteady shock interactions and short time integrations. Any numerical dissipation inherent in the scheme, even for high resolution shockcapturing schemes that maintain their high-order accuracy in smooth regions (e.g., fifth- and seventh-order WENO schemes (WENO5 and WENO7) ), ${ }^{20}$ will be compounded over long time integration leading to smearing of turbulence fluctuations to unrecognizable forms. The current trends in the containment of numerical dissipation in DNS and LES of turbulence with shocks are summarized in Yee \& Sjögreen and Yee et al. ${ }^{3,4,22}$ See the cited references for details on these current trends. Here, the performance of the high-order nonlinear filter schemes with preprocessing and postprocessing steps in conjunction with the use of a high-order non-dissipative spatial base scheme $e^{4,22}$ is briefly summarized.

Before the application of a high-order non-dissipative spatial base scheme, the preprocessing step to improve stability had split inviscid flux derivatives of the governing equation(s) in the following three ways, depending on the flow types and the desire for rigorous mathematical analysis or physical argument. 
- Entropy splitting of Olsson \& Oliger $^{23}$ and Yee et al.: ${ }^{10,24}$ The resulting form is non-conservative and the derivation is based on entropy norm stability with numerical boundary closure for the initial value boundary problem.

- The system form of the Ducros et al. splitting: ${ }^{11}$ This is a conservative splitting and the derivation is based on physical arguments.

- Tadmor entropy conservation formulation for systems (Sjögreen \& Yee ${ }^{12}$ ): The derivation is based on mathematical analysis. It is a generalization of Tadmor's entropy formulation to systems and has not been fully tested on complex flows.

After the application of a non-dissipative high-order spatial base scheme on the split form of the governing equation(s), in order to further improve nonlinear stability from the non-dissipative spatial base scheme, the postprocessing step of Yee \& Sjögreen, ${ }^{4}{ }^{25}$ Sjögreen \& $Y^{26}{ }^{26}$ is applied to nonlinearly filter the solution by a dissipative portion of a high-order shock-capturing scheme with a local flow sensor. These flow sensors provide locations and amounts of built-in shock-capturing dissipation that can be further reduced or eliminated.

To be more precise, the idea of these nonlinear filter schemes for turbulence with shocks is that, instead of solely relying on very high-order high-resolution shock-capturing methods for accuracy, the filter schemes $^{10,25-27}$ take advantage of the effectiveness of the nonlinear dissipation contained in good shockcapturing schemes as stabilizing mechanisms at locations where needed. Such a filter method consists of two steps: a full time step using a spatially high-order non-dissipative base scheme, followed by a postprocessing filter step. The postprocessing filter step consists of the products of wavelet-based flow sensors and nonlinear numerical dissipations. The flow sensor is used in an adaptive procedure to analyze the computed flow data and indicate the location and type of built-in numerical dissipation that can be eliminated or further reduced. The nonlinear dissipative portion of a high-resolution shock-capturing scheme can be any TVD, MUSCL, ENO, or WENO scheme. By design, the flow sensors, spatial base schemes and nonlinear dissipation models are standalone modules. Therefore, a whole class of low dissipative high-order schemes can be derived with ease. Unlike standard shock-capturing and/or hybrid shock-capturing methods, the nonlinear filter method requires only one Riemann solve per dimension per time step, independent of time discretizations. The nonlinear filter method is more efficient than its shock-capturing method counterparts employing the same order of the respective methods. See ${ }^{4,6,22}$ for the recent improvements of the work ${ }^{10,25-27}$ that are suitable for a wide range of flow speed with minimal tuning of scheme parameters. For all the computations shown, the Ducros et al. splitting is employed since a conservative splitting is more appropriate if one does not know if the subject flow is shock-free or turbulence with shocks (see e.g., Section 2.2.2). Some attributes of the high-order filter approach are:

- Spatial Base Scheme: high-order and conservative (no flux limiter or Riemann solver)

- Physical Viscosity: Contribution of physical viscosity, it it exists, is automatically taken into consideration by the base scheme in order to minimize the amount of numerical dissipation to be used by the filter step.

- Efficiency: One Riemann solve per dimension per time step, independent of time discretizations (less CPU time and fewer grid points than their standard shock-capturing scheme counterparts).

- Accuracy: Containment of numerical dissipation via a local wavelet flow sensor.

- Well-balanced scheme: These nonlinear filter schemes are well-balanced schemes for certain chemical reacting flows. ${ }^{6}$

- Parallel Algorithm: Suitable for most current supercomputer architectures.

\section{Variable High-Order Multiblock Overset Grid Methods ${ }^{1,9}$}

For over two decades, second- and third-order shock-capturing schemes employing time-marching to the steady state have enjoyed much success in simulating many transonic, supersonic and hypersonic steady aeronautical flows containing strong shocks. In the presence of mixed steady and unsteady multiscale viscous flows, low order (third-order or lower) time-accurate methods are not effective in accurately simulating, 
e.g., unsteady turbulent fluctuation containing shocklets. At the same time, high-order schemes with good unsteady shock-capturing capability suffer from the inability to converge to the proper steady shocks effectively. Attempts to improve the convergence rate of high-order methods for strong steady shocks involve order reduction near steep gradient regions or added numerical dissipation of the scheme in the vicinity of the shocks, thus degrading the true order of the scheme in other parts of the flow. Although extreme grid refinement in conjunction with low order schemes can be used on the unsteady turbulence part of the flow field, increases in CPU time, and instability and stiffness of the overall computations are inevitable. A method to effectively overcome these difficulties for mixed steady and unsteady viscous flows is a multiblock overset grid with a different order and different type of numerical scheme on different blocks.

Stable SBP (summation-by-parts) energy norm numerical boundary procedures ${ }^{28}$ for high-order central spatial schemes are employed at physical boundaries. Second-, third- and fourth-order Lagrangian interpolations are options in the solver ADPDIS3D to be used for interpolating grid point values among the block overlapping regions. ${ }^{29}$ For stability, in most of the computations, a second-order interpolation is preferred. Matching high-order spatial scheme with viscous and metric evaluations with freestream preservation are used in the presence of physical viscosity and curvilinear grids, ${ }^{7}$ respectively. The multiblock option can, e.g., easily accommodate low order shock-capturing schemes in regions of steady shocks and high-order schemes in regions containing unsteady turbulence and shocklets. See ${ }^{1,9}$ for details.

An important application for the proposed solver is to simulate blunt body space vehicles at hypersonic speeds with strong steady or nearly steady bow shocks and possible complex turbulence/shocklet interaction near the shoulder of the space vehicles and wake region at different angles of attack. It is also important for the study of the leading edge heat shield due to surface irregularities and/or isolated surface singularities such as very small openings. Another application is in numerical modeling of the heliosphere, space weather fore-castings, supernova explosions and inertial confinement fusion.

\title{
III. Overset grid numerical results for chemical nonequilibrium flows
}

Before embarking on multiscale problems containing mixed steady and unsteady shock/turbulence interaction, we first illustrate several simple blunt body test cases to validate the high-order overlapping approach. The test cases were intentionally chosen to contain strong bow shock without mixed unsteady components in the flow. Furthermore, in order to validate the proposed variable high-order multiblock overlapping grid methods, as an illustration, only two different orders of schemes are used.

\section{A. A 2D chemical nonequilibrium flow past a cylinder}

A 2D testcase simulating high speed air flow around a $1[\mathrm{~m}]$ radius cylinder has been chosen for the inviscid and viscous numerical experiments. The free stream and wall conditions (for the viscous case) are given in Table 1. This testcase was computed by Peter Gnoffo (private communication) and further studied by Xiaowen Wang from the UCLA SciDAC team. The physico-chemical model used in the present work does not consider thermal nonequilibrium as in Xiaowen Wang's study, but uses more sophisticated and computationally expensive thermodynamic and transport properties (see Section II.A for details) as opposed to energy fitting polynomials and mixture rules. The chemical reaction rate coefficients for characterizing the neutral air mixture are taken from ${ }^{30}$ by neglecting reactions involving ions and electrons. Here, the

\begin{tabular}{cccccc}
\hline$M_{\infty}$ & $\rho_{\infty}\left[\mathrm{kg} / \mathrm{m}^{3}\right]$ & $U_{\infty}[\mathrm{m} / \mathrm{s}]$ & $T_{\infty}[K]$ & $T_{w}[K]$ & $R e_{\infty}$ \\
\hline 17.64 & 0.0001 & 5000 & 200 & 553.3 & 37634.8 \\
\hline
\end{tabular}

Table 1. Free stream and wall conditions for Gnoffo's testcase.

performance of TVD, WENO5 and WENO7 on the single block and overset meshes for the inviscid case is discussed first. Then, some results with mixed WENO5 and sixth-order filter central scheme on the overset mesh are presented. Next, the viscous case on both a single block and overset meshes using the TVD scheme is considered. Finally, a simulations of higher-order filter schemes for the viscous case is included. Since this is a time-accurate approach, the residual tracking has not been used as convergence criterion. However, simulations are computed for long enough (typically up to 500,000 iterations and 1,000,000 iterations for inviscid and viscous cases respectively, using a CFL number up to 0.8 , depending on the case) to allow the

\author{
7 of 16 \\ American Institute of Aeronautics and Astronautics
}


flow to be fully established.

\section{Inviscid chemical nonequilibrium: TVD scheme versus WENO schemes}

A reference single block case has been run on two meshes: coarse (61 x 129 nodes) and fine (4 times finer in both directions). Figure 1 shows a comparison between TVD, and WENO5-LF schemes on both grids in terms of stagnation line temperature profile. As the grid spacing is decreased, the shock becomes steeper (especially for the TVD scheme) and the peak temperature increases about $6 \%$ for both schemes (see Fig. 2). On the fine mesh WENO and TVD solutions are indistinguishable, indicating that grid convergence has been achieved. On both coarse and fine meshes, WENO5-LF delivers a clean oscillation-free solution on all flow quantities. The use of WENO7 or WENO9 schemes, as reported in, ${ }^{31}$ does not yield significant accuracy improvements for the specific test case compared to WENO5. Moreover, well balanced WENO-LF schemes ${ }^{6}$ give results which are almost indistinguishable from the corresponding baseline schemes, at least for this case. The reason is partly due the fact that the improvement found in higher-order WENO schemes is away from the shock, and partly due to the considered blunt body test case with the bow shock dominating the flow structure. The advantage of higher-order schemes would only comes into play for flows containing more complex unsteady structures.

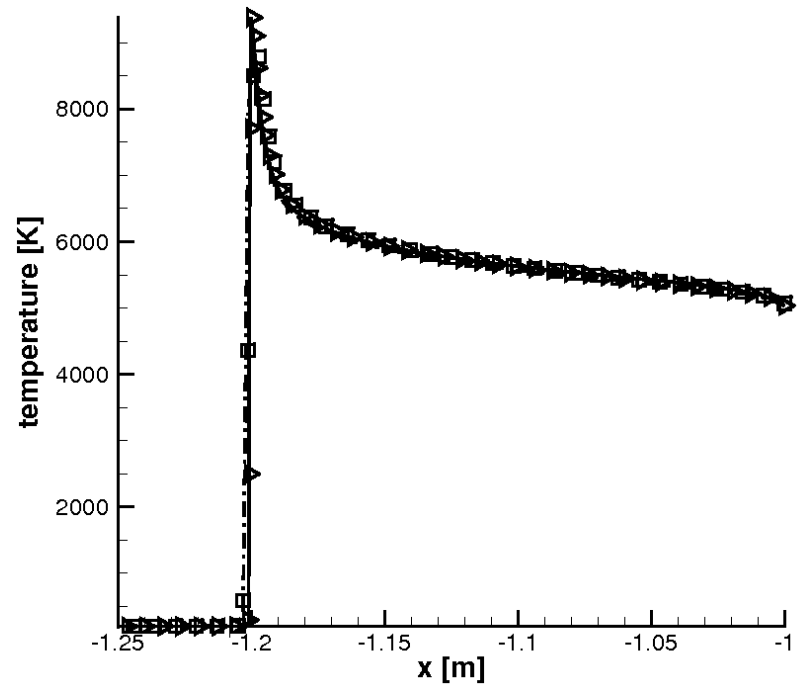

Figure 1. Stagnation line temperature: TVD on coarse (dashdot line) and fine mesh (solid line), WENO5-LF on coarse (squares) and fine mesh (right triangles).

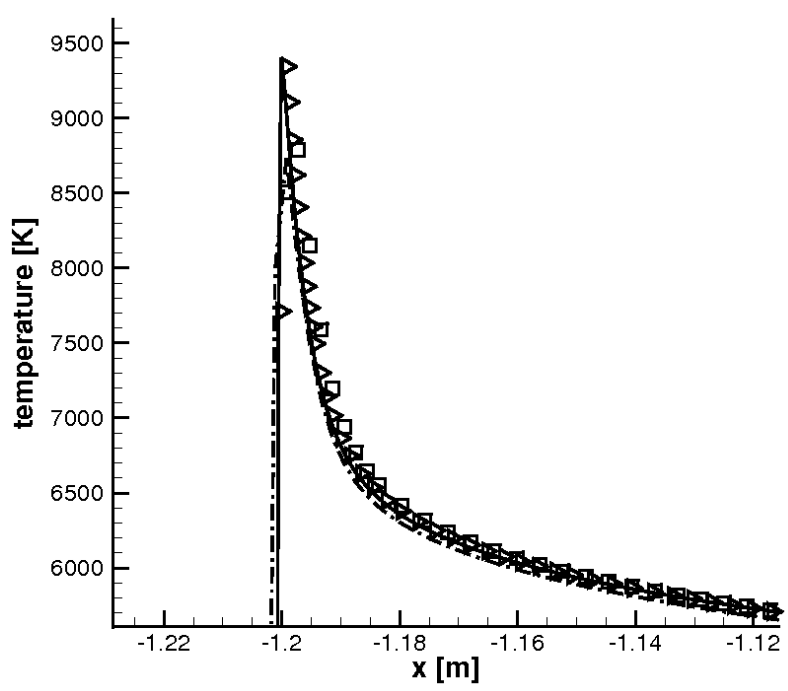

Figure 2. Zoom around the peak temperature: TVD on coarse (dashdot line) and fine mesh (solid line), WENO5-LF on coarse (squares) and fine mesh (right triangles).

The overset grid simulation consists of three-blocks with its upper half shown in Fig. 3. The three blocks consist of background (123x122 nodes), shock (201x40 nodes), and body (123x40 nodes) blocks. For validation purposes, even though the chosen mesh includes a much larger than needed background block, the total number of grid points in the region of interest is about $60 \%$ of the total. In particular, there are about 70 points along the direction normal to wall inside the shock layer. The flow solution in terms of Mach number contours is shown in Fig. 4. Using the same scheme on all blocks, the TVD, WENO5-LF and WENO7-LF results are almost indistinguishable. In particular, a smooth solution transition from one block to another can be observed, even in the postshock region where all three mesh blocks overlap. Figure 5 compares the temperature stagnation line profiles by the three schemes. A negligible difference in the shock standoff distances between TVD and WENO and a $2 \%$ higher temperature peak prediction by WENO can be observed. Finally, temperature stagnation profiles computed by WENO5-LF, WENO7-LF, WENO5-LFWB and WENO7-LF-WB are shown in Fig. 6. As expected, there is no visible difference in results among WENO5-LF, WENO5-LF-WB and WENO7-LF, except for WENO7-LF, which tends to predict a slightly higher temperature. 


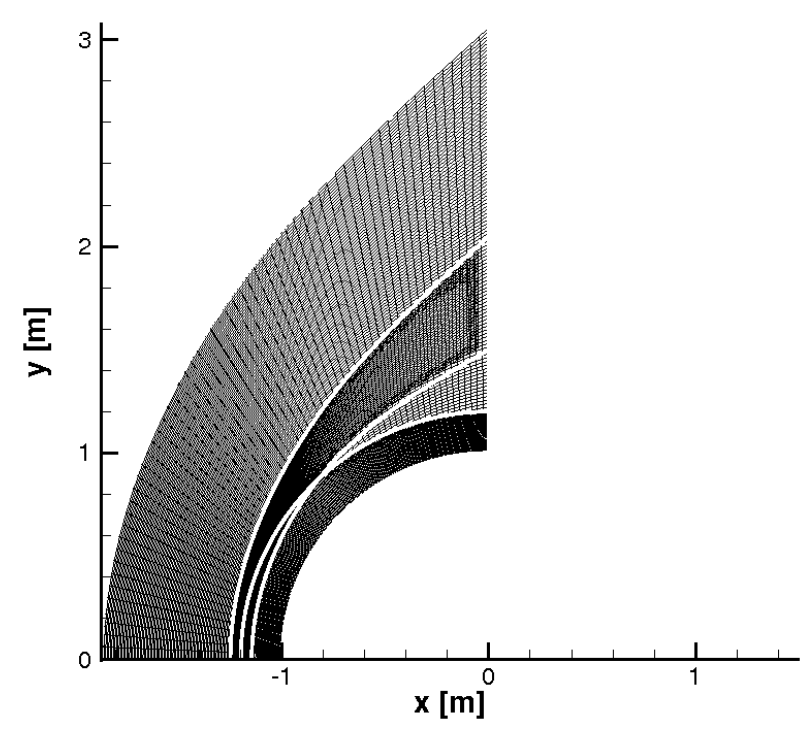

Figure 3. Upper half of the three-block overset mesh used for Gnoffo's testcase. Boundaries for shock and body blocks are highlighted in white.

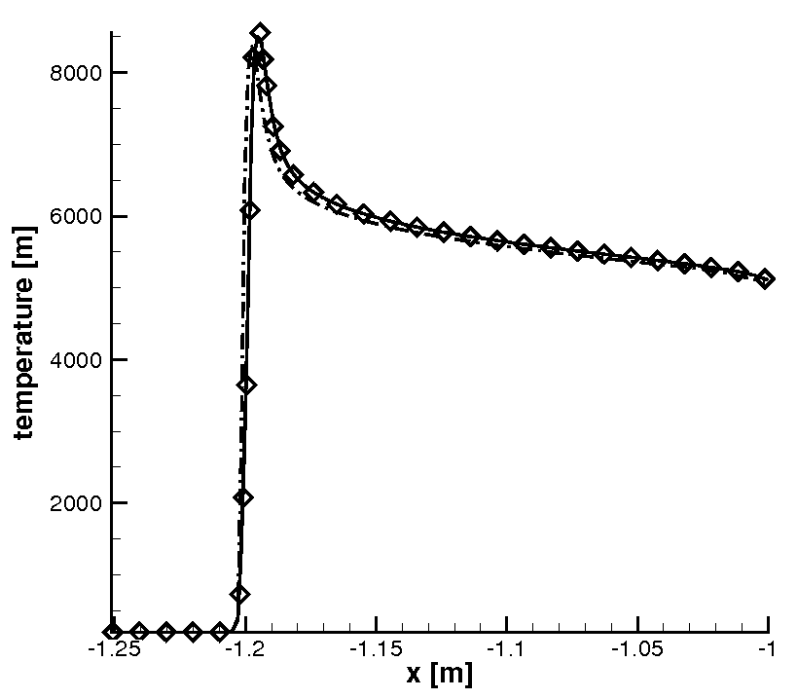

Figure 5. Stagnation line temperature on the threeblock overset mesh: TVD (dashdot line), WENO5-LF (solid line), WENO7-LF (right diamonds).

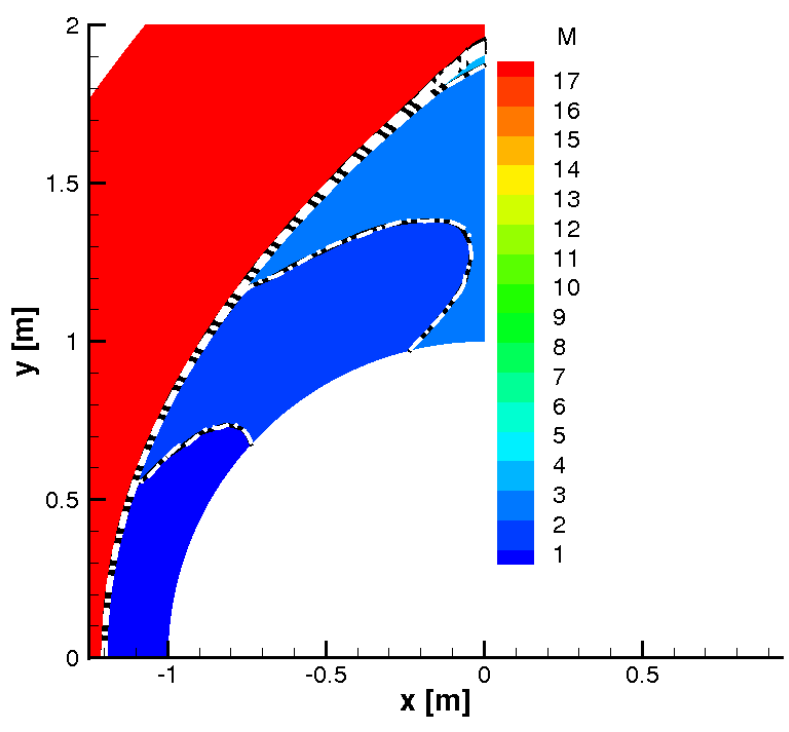

Figure 4. Mach contours on the overset mesh: TVD (contours), WENO5-LF (black isolines), WENO7-LF (white dashdot isolines). Part of the background block has been left out.

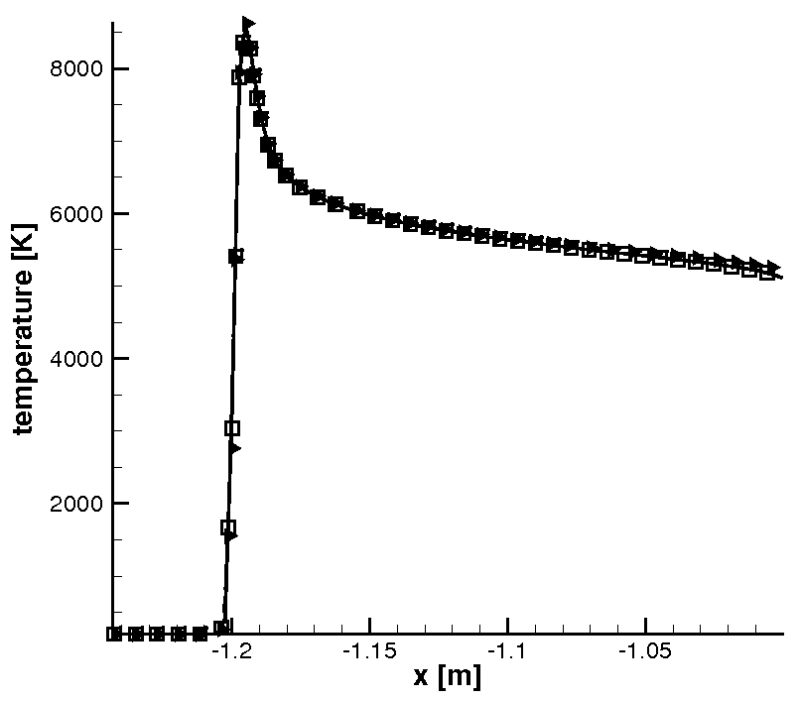

Figure 6. Stagnation line temperature on the overset mesh: WENO5-LF (solid line), WENO5-LFWB(dashdot line), WENO7-LF (squares), WENO7LF-WB (right squares). 


\section{Inviscid chemical nonequilibrium: Mixed WENO5 and sixth-order central filter scheme (WENO5fi)}

Results using variable high-order methods have been computed on two overset meshes, one fine and one coarse, with a slightly larger shock block than in Fig. 3. The finer mesh has the same number of grid points as the mesh in Fig. 3, whereas the coarser mesh has half the number of grid points. WENO5-LF was used for the shock block, while a sixth-order central discretization with dissipative portion of the corresponding WENO scheme as a non-linear filter (WENO5fi) $)^{2,3}$ was used on the body and background blocks. Figures 7 and 8 illustrate the temperature isolevels and stagnation line profiles for the two meshes. The shock and the overall flowfield are well captured even on the coarse overset mesh. Again a smooth transition between blocks has been achieved.

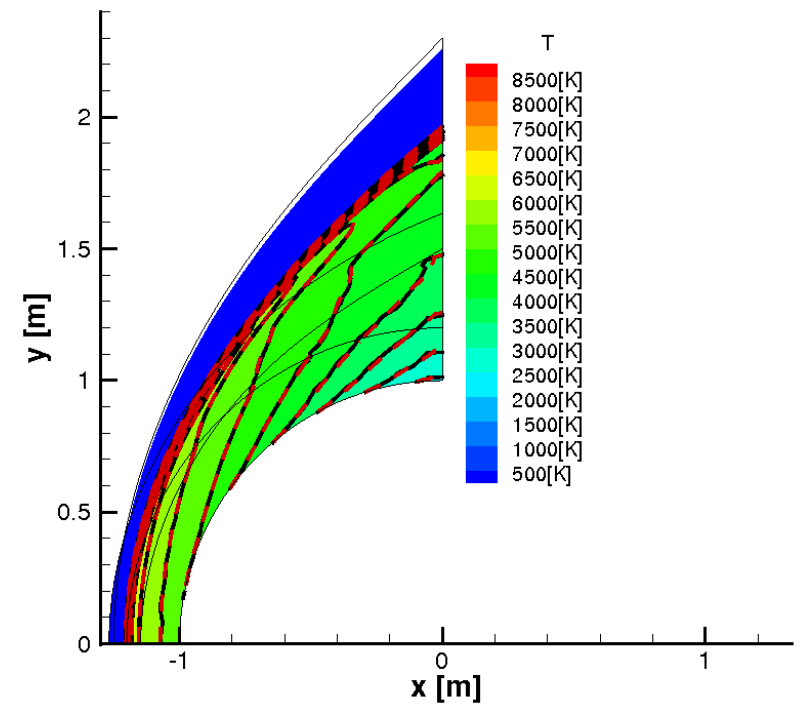

Figure 7. Temperature isolevels (in K) on the coarse (red dashed isolines) and fine (black solid isolines) overset meshes: sixth-order central filtered (WENO5fi) is applied on body and background blocks, WENO5-LF on the shock block. Part of the background block has been left out.

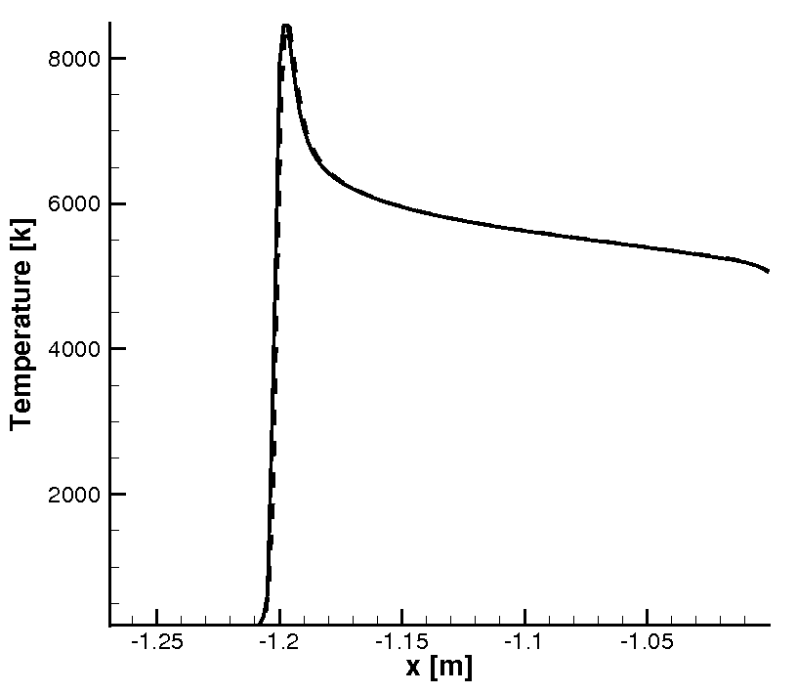

Figure 8. Temperature stagnation line profiles on the coarse (dashed lines) and fine (solid lines) overset meshes: sixth-order central filtered (WENO5fi) is applied on body and background blocks, WENO5-LF on the shock block.

Figures 9 and 10 show pressure and temperature fields, respectively, computed on the fine mesh by the variable high-order method for the three different cases WENO5-LF, WENO5-LF-WB and TVD schemes on the shock block. While their pressure isolines are indistinguishable, some small differences can be identified in the temperature isolines between WENO schemes and TVD within the shock block. The difference increases while moving far from the stagnation region where the mesh is less aligned with the flow.

\section{Viscous chemical nonequilibrium: TVD scheme}

The interested reader is referred to ${ }^{31}$ for a preliminary validation of Gnoffo's testcase in viscous conditions on single block grids. This subsection presents instead a comparison of the same viscous testcase on three different grid configurations: a) a single fine grid with $244 \times 516$ points, b) the overset grid shown in Fig. 3 , and c) a fine overset grid. The fine overset grid has $201 \times 120$ points in the shock block and $123 \times 240$ points in the boundary layer block. For consistency with the reference single block grid solution, no stretching has been applied in the direction normal to the wall, where the corresponding grid spacings are 0.0004 for the single grid, 0.005 for the overset grid in Fig. 3, and 0.0008 for the finer overset grid. The results are shown in Figs. 11 and 12 in terms of pressure and temperature and were all computed by the Harten-Yee TVD scheme. The pressure isolines/levels and shock standoff distance are perfectly matching between the fine single and multiblock meshes. When refining the overset mesh, the solution in terms of temperature isolines consistently gets closer to the fine single block mesh case, but some differences persist in the boundary layer region, probably due to the nearly twice finer normal spacing in the single block grid. 


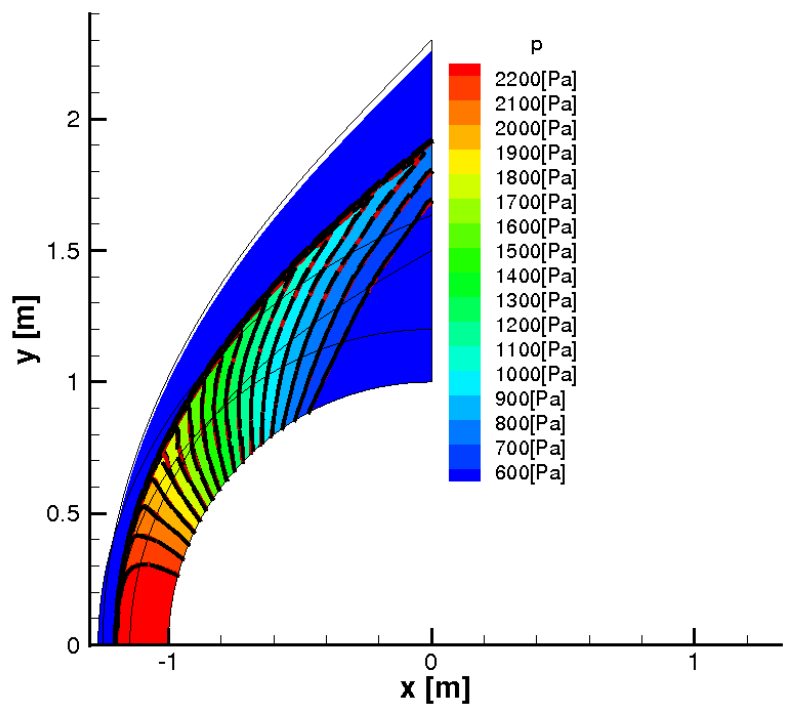

Figure 9. Pressure isolevels (in $\mathbf{P a}$ ) on the overset mesh: sixth-order central filtered (WENO5fi) is applied on body and background block, WENO5-LF (black solid isolines), WENO5-LF-WB (red dashed isolines), TVD (black long dashed isolines) on the shock block.

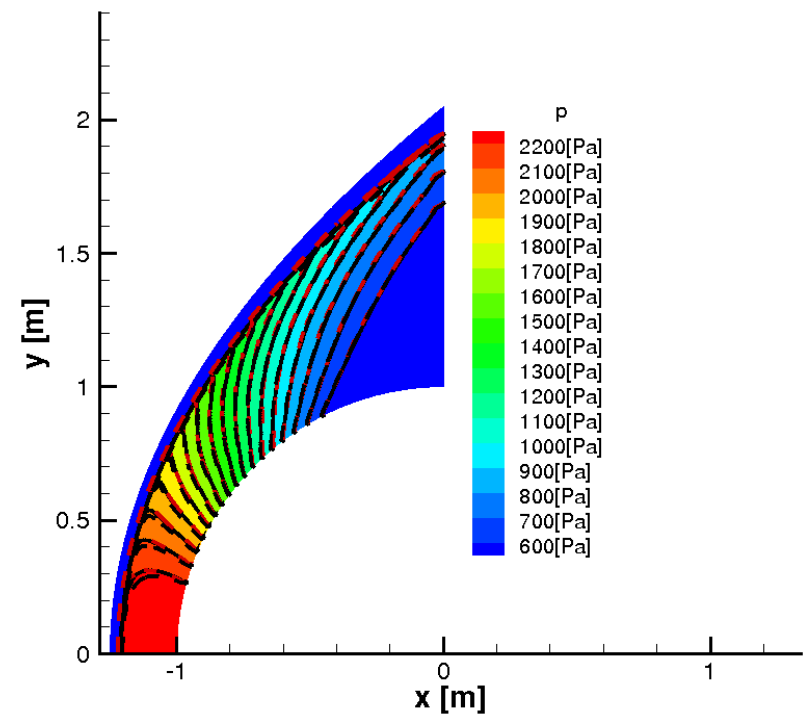

Figure 11. Pressure field (in Pa): single block mesh (black solid isolines and contours), coarse (red dashed isolines) and fine (black dashed isolines) overset mesh.

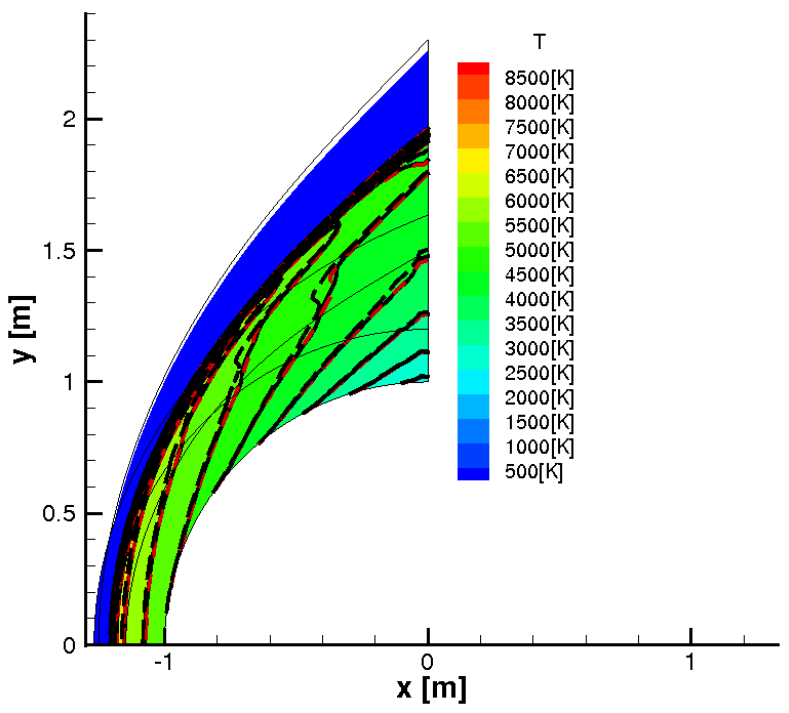

Figure 10. Temperature isolevels (in K) on the overset mesh: sixth-order central filtered (WENO5fi) is applied on body and background block, WENO5-LF (black solid isolines), WENO5-LF-WB (red dashed isolines), TVD (black long dashed isolines) on the shock block.

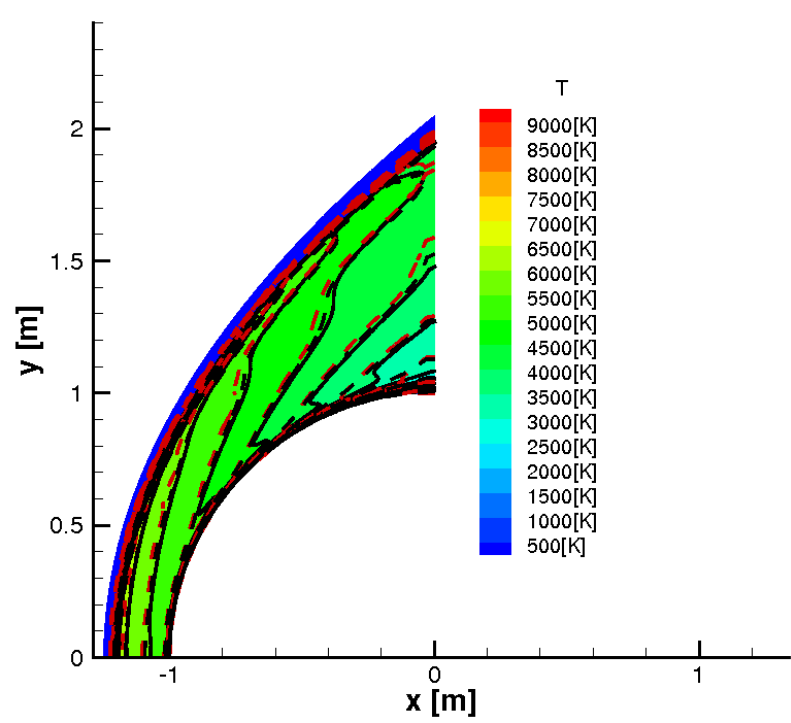

Figure 12. Temperature field (in K): single block mesh (black solid isolines and contours), coarse (red dashed isolines) and fine (black dashed isolines) overset mesh. 


\section{Viscous chemical nonequilibrium: Mixed TVD and fourth-order central filter schemes}

Results using variable high-order methods have been computed on a different three-block overset mesh for which the boundary layer block does not overlap with the shock block (see Fig. 13). Figure 14 shows the temperature obtained with a mixed approach, where the second-order TVD scheme is used on the shock grid and the fourth order centered approximation, filtered nonlinearly with the dissipative portion of the TVD scheme (D04+TVDfi) in conjunction with Ducros et al. splitting a preprocessing step discussed in Section $\mathrm{I}$, is used on the boundary layer grid.

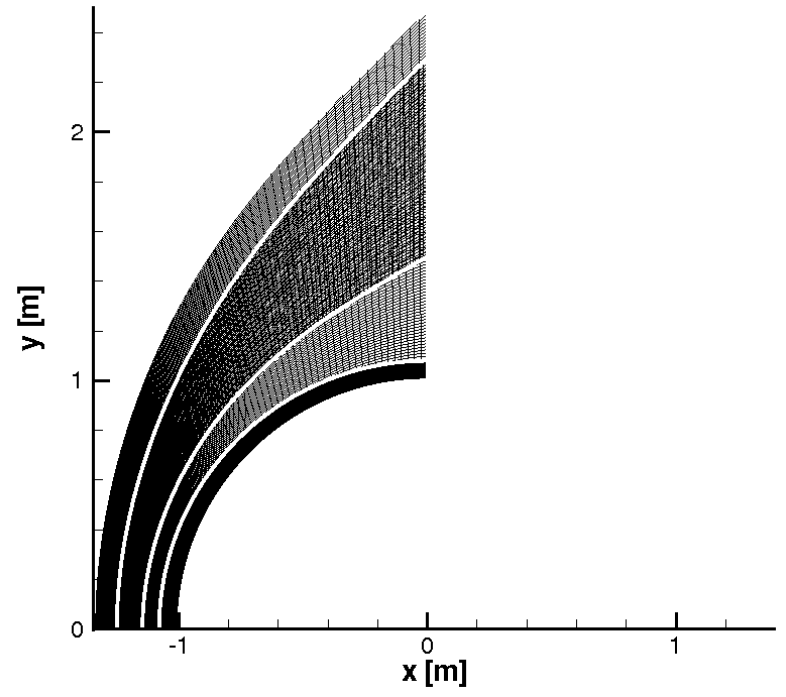

Figure 13. Upper half of the three-block overset mesh used for the mixed order viscous computation. Boundaries for shock and body blocks are highlighted in white on the boundary layer grid.

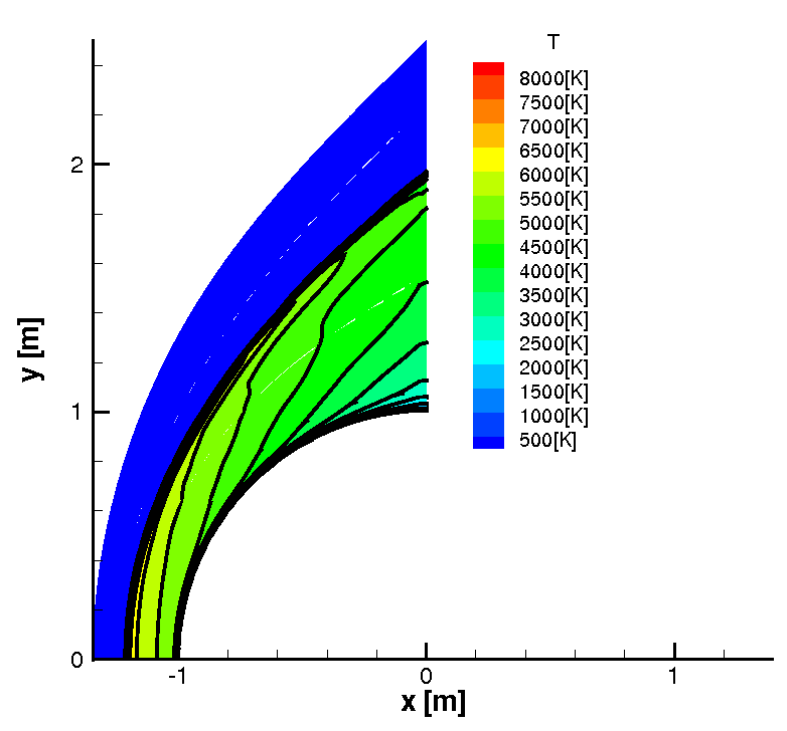

Figure 14. Temperature contours and isolines with fourth-order central with TVD filter.

Figure 15 show the close up boundary-layer grid overlapping with the background grid. Figure 16 displays iso-density contours of the solution by the TVD scheme on the overset grid shown in Fig. 12. Figures 17-18 show solutions obtained with different fourth-order schemes on the boundary layer grid, while keeping the TVD scheme on the other grids. Figure 17 shows the solution with the fourth-order spatial central scheme and sixth-order constant linear dissipation (D04+AD6) on the boundary grid. Figure 18 shows the result by the same fourth-order central spatial scheme on the boundary grid but with the nonlinear TVD filter as a postprocessing step (D04ss+TVDfi) instead of the sixth-order linear dissipation. D04ss+TVDfi also employs the Ducros et al. splitting as a preprocessing step in previous section I. The solution shown appears to have been converged (with several hundreds thousand of iterations) with inaccurate solution at the high aspect ratio overlapping boundary grid region due to insufficient grid points. It is expected that one or two level of grid refinement will overcome the inaccuracy problem. The grid refinement study will be included in a forthcoming paper.

\section{B. 3D chemical nonequilibrium example: CEV computation}

Improved CFD predictability of future CEV afterbody flowfields in various flight conditions are of great importance for future aerospace explorations. In light of the fact that future CEV has increased in size and weight, and consequently has higher Reynolds numbers over the previously considered configurations, the ability to better characterize the base heating and the role of transition and turbulence in future aerothermodynamic design ${ }^{32,33}$ remains a pacing item for aerothermodynamicists. Here, the preliminary investigation on a CEV-like geometry started in $^{1}$ is continued but in conditions (see Table 2) taken from, ${ }^{34}$ and corresponding to the maximum Reynolds number point in the reentry trajectory of the FIRE II experiment.

The first step of the investigation consists of a time-accurate Navier-Stokes computation without any 


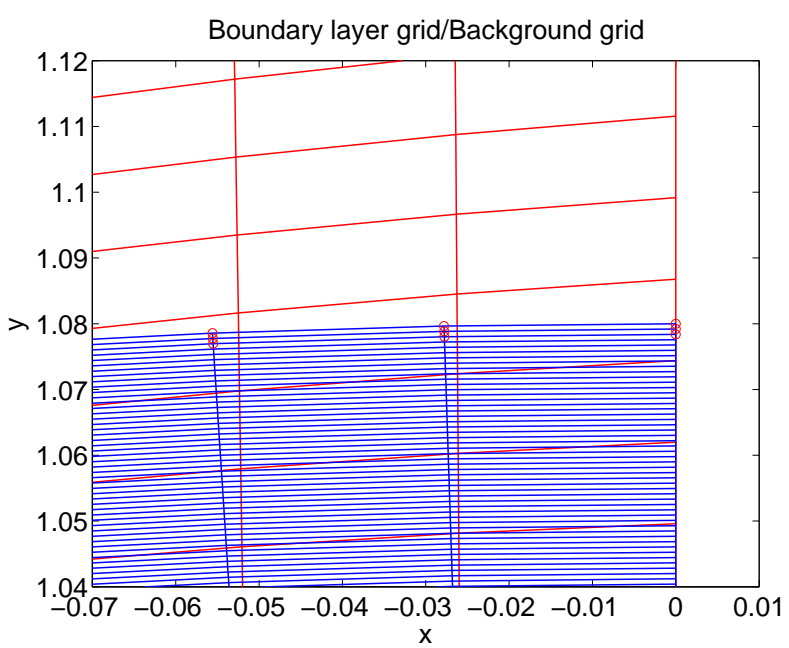

Figure 15. Boundary layer grid at one segment of the boundary.

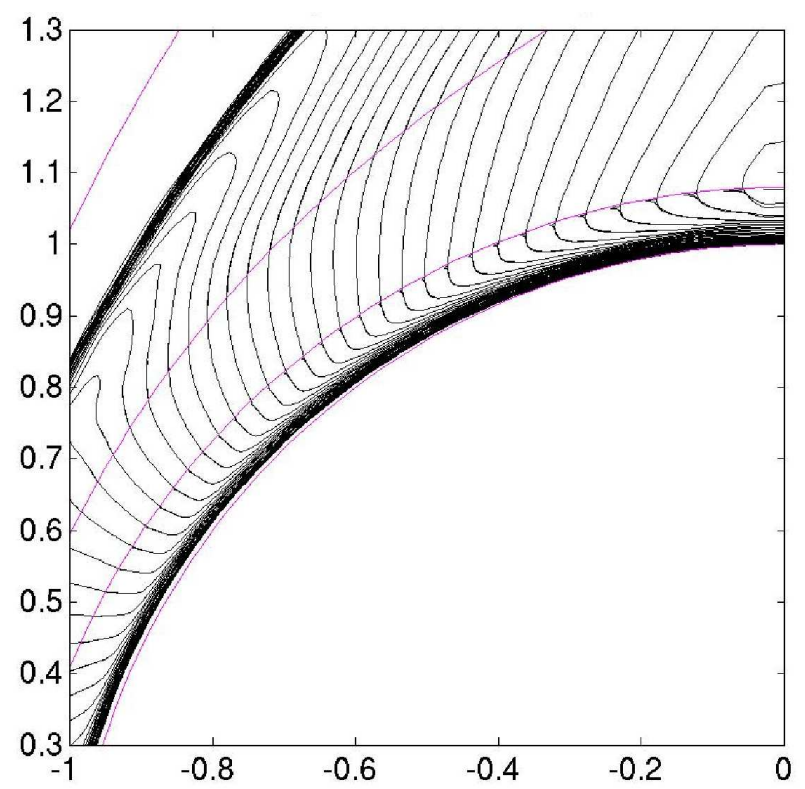

Figure 17. Density isolines when D04+AD6 is used in the boundary layer block.

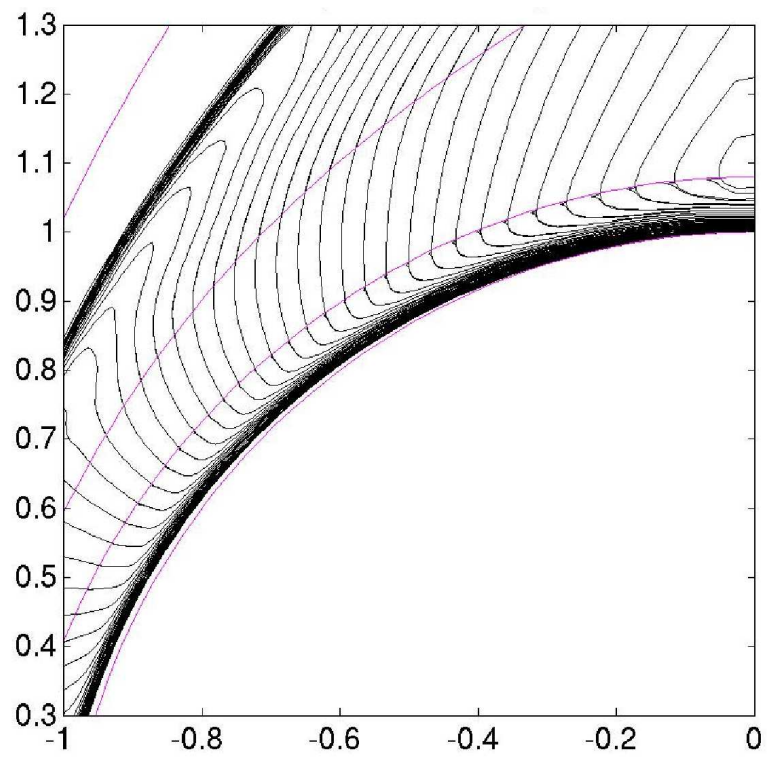

Figure 16. Density isolines when second-order TVD scheme is used in the boundary layer block.

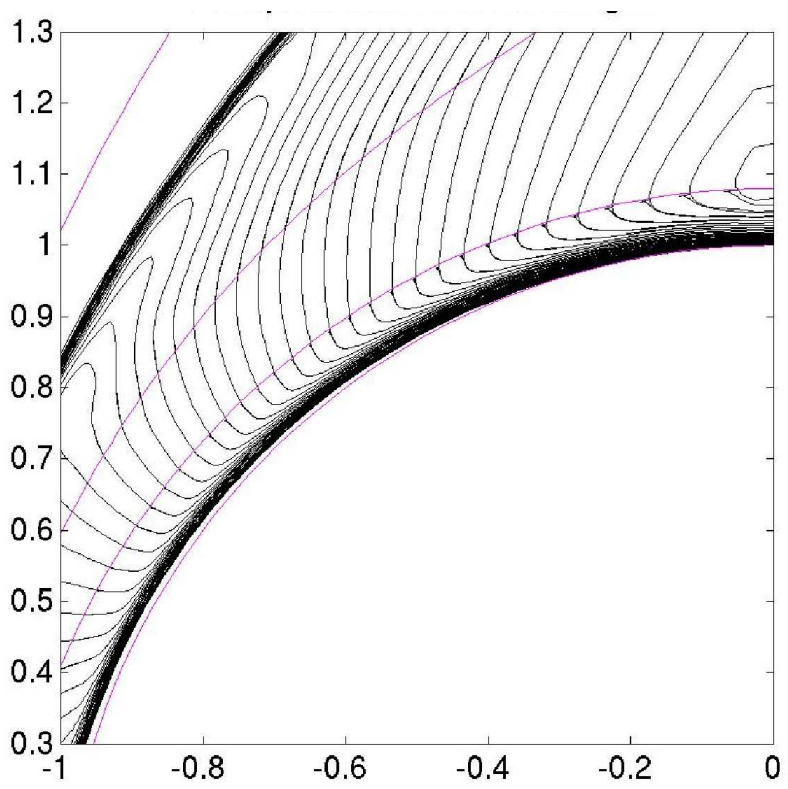

Figure 18. Density isolines when D04ss+TVDfi is used in the boundary layer block. 
turbulence model but including chemical nonequilibrium effects. The same neutral five-species model used for the $2 \mathrm{D}$ case is utilized here, with reaction rate coefficients derived from. ${ }^{30}$ Thermodynamics and transport properties are described in Section A. For low enough Reynolds number and with fine enough grid to resolve all scales, the simulation could be considered as a DNS computation.

\section{Viscous flow: TVD scheme}

A six-block 3D viscous overset mesh consisting of $26.5 \mathrm{M}$ nodes was used as a first step in the investigation. In order to keep the computational cost affordable, the size of the first cell on the wall has been set to 0.003 [m]. A snapshot of the overset mesh, featuring all six blocks around and on the capsule, is depicted in Fig. 19. With the purpose of obtaining a benchmark flow field, the TVD scheme was applied to four blocks and a first-order Roe scheme was applied on the two blocks containing the shock. The flow conditions for this testcase are listed in Table 2.

\begin{tabular}{cccccccc}
\hline$h[\mathrm{~km}]$ & $M_{\infty}$ & $\rho_{\infty}\left[\mathrm{kg} / \mathrm{m}^{3}\right]$ & $U_{\infty}[\mathrm{m} / \mathrm{s}]$ & $\alpha\left[^{\circ}\right]$ & $T_{\infty}[K]$ & $T_{w}[K]$ & $R e\left[10^{6}\right]$ \\
\hline 35 & 16 & 0.0082 & 5000 & 0 & 237 & 553.3 & 1.76 \\
\hline
\end{tabular}

Table 2. Free stream and wall conditions for the CEV simulation.

Figure 20 shows that all the typical features of this kind of flow are well detected (see a perfect gas solution from ${ }^{3,34}$ for a qualitative comparison). After undergoing a severe compression through the bow shock, the flow heats up to about $6000 \mathrm{~K}$ in the stagnation region. The strong expansion around the aft causes the temperature to drop considerably and the laminar boundary layer to separate at the beginning of the conical afterbody. A thick rake of shear layers forms, enclosing a large recirculation region which extends up to the neck region about one and a half capsule diameters further, where a weak recompression shock wave forces the flow to return parallel to the axis.

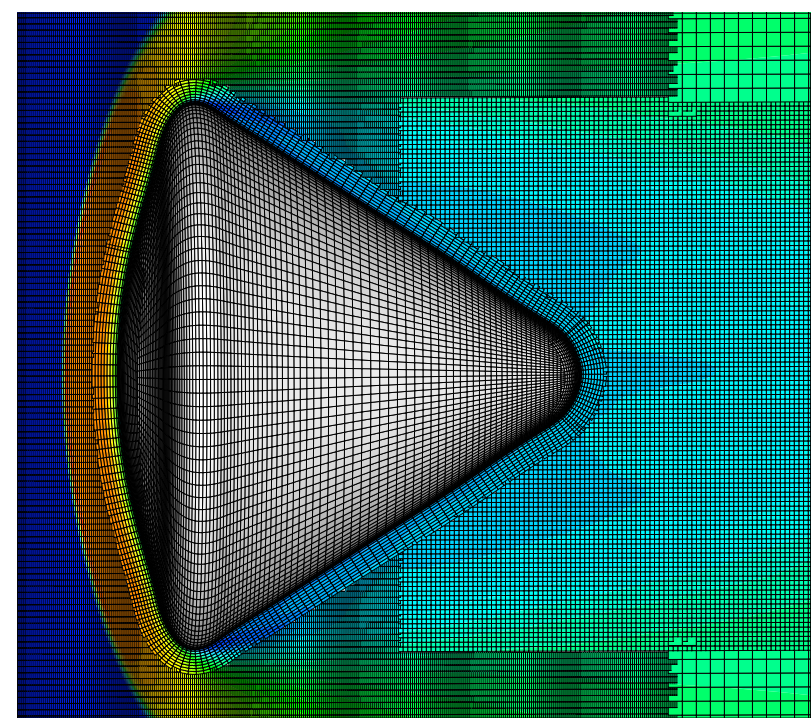

Figure 19. View on the overset mesh on the CEV capsule surface and on the $\mathrm{x}-\mathrm{z}$ symmetry plane.

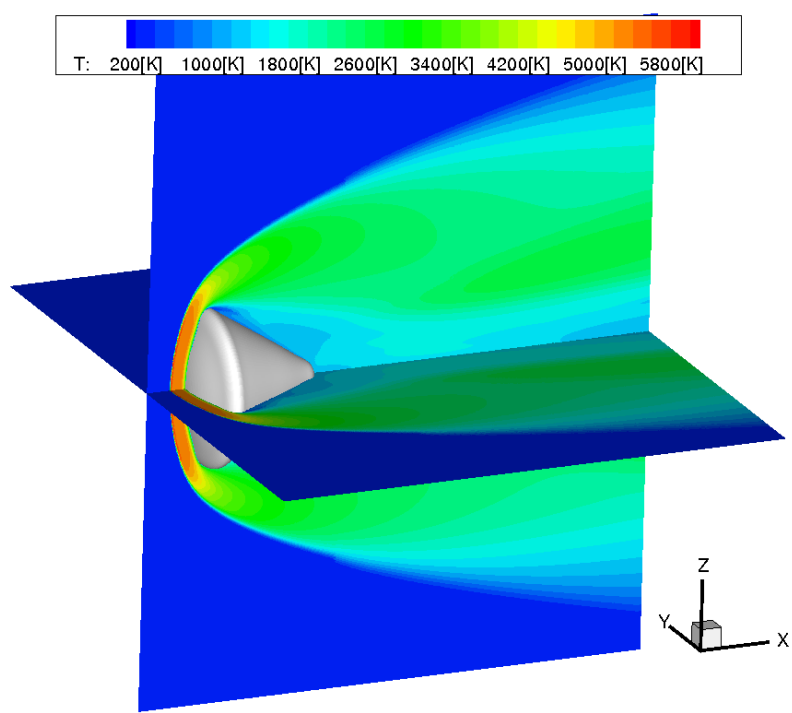

Figure 20. Temperature contours for CEV capsule.

\section{Conclusions}

In the present paper, variable high-order methods have been applied to the simulation of hypersonic flows in chemical nonequilibrium on multiblock overlapping meshes. In order to validate the time-accurate nonequilibrium flow implementation, a time-accurate approach has been conducted on 2D and 3D inviscid and viscous chemical nonequilibrium laminar lows with strong shocks before embarking on multiscale problems containing both steady and unsteady shock/turbulence interactions. All the considered test cases are 
laminar flows with a strong steady bow shock. A fourth-order or sixth-order central space discretizations has been successfully combined with upwind TVD or WENO schemes for 2D inviscid and viscous cases, where the lower order has been confined only to the block including the bow shock. The variable high-order method has been further validated in the case of the high-speed flow over the 3D CEV space vehicle, under realistic flight conditions. The results of a preliminary viscous computation with TVD schemes has been shown and will be the basis for future analysis where high-order filter schemes will be used away from the shock to better resolve critical flow features such as the laminar boundary layer up to the capsule shoulder, the flow separation on the conical afterbody, the shock/shear interaction occurring in the neck region and the overall wake dynamics. Representative test cases with mixed steady and unsteady turbulence with strong shocks components that can benefit fully from the present high accuracy approach will be presented in forthcoming papers.

\section{Acknowledgments}

The authors wish to express their gratitude to A. Lazanoff and J. Chang of the Scientific Consultant Group, Code TN, NASA Ames and their help. The support of the DOE/SciDAC SAP grant DE-AI0206ER25796 is acknowledged. Work by the second and fourth authors was performed under the auspices of the U.S. Department of Energy by Lawrence Livermore National Laboratory under Contract DE-AC5207NA27344. Part of the work by the third author was performed under NASA Fundamental Aeronautics Hypersonic Program. Special thanks to Wei Wang, former CTR postdoc, who implemented all WENO schemes for nonequilibrium flows.

\section{References}

\footnotetext{
${ }^{1}$ Sjögreen, B. and Yee, H. C., "Variable High Order Multiblock Overlapping Grid Methods for Mixed Steady and Unsteady Multiscale Viscous Flow," Comm. Comput. Phys., Vol. 5, No. 2-4, 2009, pp. 730-744.

${ }^{2}$ Yee, H. C. and Sjögreen, B., "Nonlinear Filtering and Limiting in High Order Methods for Ideal and Non-ideal MHD," J.Sci.Comp, Vol. 27, 2006, pp. 507-521.

${ }^{3}$ Yee, H. C., Sjögreen, B., and Barone, M., "High order numerical schemes for hypersonic flow simulations," VKI Lecture Series: Course on hypersonic entry and cruise vehicles, Von Karman Institute for Fluid Dynamics, Stanfor University, Palo Alto (CA), 30 June - 3 July 2008.

${ }^{4}$ Yee, H. and Sjögreen, B., "High Order Filter Methods for Wide Range of Compressible Flow Speeds," Proceedings of ICOSAHOM 09, International Conference on Spectral and High Order Methods, Trondheim, Norway, Jun 22-26 2009.

${ }^{5}$ Yee, H. C., Sjögreen, B., and Hadjadj, A., "Comparative study of high order schemes for LES of temporally evolving mixing layers," Extended version of the paper for the Proceedings of ASTRONUM-2010, June 13-18, 2010, San Diego, Calif; submitted to J. Comp. \& Fluids.

${ }^{6}$ Wang, W., Yee, H. C., Sjögreen, B., Magin, T., and Shu, C. W., "Construction of low dissipative high-order well balanced filter schemes for non-equilibrium flows," J. Comput. Phys., 2010.

${ }^{7}$ Vinokur, M. and Yee, H., "Extension of Efficient Low Dissipative High Order Schemes for 3-D Curvilinear Moving Grids," Tech. rep., NASA TM 209598, June 2000, also appear at the Proceedings of the Frontiers of Computational Fluid Dynamics, 2002, World Scientific, D.A. Caughey \& M. Hafez editors., (2002) 129-164.

${ }^{8}$ Hadjadj, A., Yee, H. C., and Sjögreen, B., "LES of temporally evolving mixing layers by high order filter schemes," submitted to J. Comput. Phys.

${ }^{9}$ Sjögreen, B., Yee, H. C., Djomehri, J., Lazanoff, A., and Henshaw, W. D., "Parallel Performance of ADPDIS3D - A High Order Multiblock Overlapping Grid Solver for Hypersonic Turbulence," Parallel CFD, Moffett Field (CA), May 18-22 2009.

${ }^{10}$ H.C. Yee, M. V. and Djomehri, M., "Entropy Splitting and Numerical Dissipation," J. Comput. Phys., Vol. 162, 2000, pp. 33-81.

${ }^{11}$ Ducros, F., Laporte, F., Soulères, T., Guinot, V., Moinat, P., and Caruelle, B., "High-Order Fluxes for Conservative Skew-Symmetric-like Schemes in Structured Meshes: Application to Compressible Flows," J. Comput. Phys., Vol. 161, 2000, pp. 114-139.

${ }^{12}$ Sjögreen, B. and Yee, H., "On Skew-Symmetric Splitting of the Euler Equations," Proceedings of the EUNUMATH-09 Conference, June 29 - July 22009.

${ }^{13}$ Henshaw, W. D., "Ogen: An Overlapping Grid Generator for Overture," Research Report UCRL-MA-132237, Lawrence Livermore National Laboratory, 1998.

${ }^{14}$ Brown, D. L., Henshaw, W. D., and Quinlan, D. J., "Overture: Object-oriented Tools for Solving PDEs in Complex Geometries," .

${ }^{15}$ Wang, W., Shu, C. W., Yee, H. C., and Sjögreen, B., "High-order well balanced schemes and applications to nonequilibrium flows," J. Comput. Phys., Vol. 228, 2009, pp. 6682-6702.

${ }^{16}$ Abeele, D. V., An Efficient Computational Model for Inductively Coupled Air Plasma Flows under Thermal and Chemical Non-Equilibrium, Ph.D. thesis, Katholieke Universiteit Leuven, Chaussée de Waterloo, 72, 1640 Rhode-St-Genèse, Belgium, Nov. 2000.
} 
${ }^{17}$ Magin, T. and Degrez, G., "Transport Algorithms for Partially Ionized and Unmagnetized Plasmas," Journal of Computational Physics, Vol. 198, 2004, pp. 424-449.

${ }^{18}$ Hirschfelder, J. O., Curtiss, C. F., and Bird, R. B., Molecular theory of gases and liquids, John Wiley and Sons, New York, 1967.

${ }^{19}$ Yee, H. C., "A class of high-resolution explicit and implicit shock-capturing methods," VKI lecture series 1989-04, NASA TM-101088, March 1989.

${ }^{20}$ Jiang, G. S. and Shu, C. W., "Efficient Implementation of Weighted ENO Schemes," J. Comput. Phys., Vol. 126, 1996, pp. 202-228.

${ }^{21}$ Stuart, A. and Humphries, A., Dynamical Systems and Numerical Analysis, Cambridge Monographs on Applied and Computational Mathematics, 1998.

${ }^{22}$ Yee, H. and Sjögreen, B., "Local Flow Sensors in Controlling Numerical Dissipations for a Wide Spectrum of Flow Speed and Shock Strength," in preparation.

${ }^{23}$ Olsson, P. and Oliger, J., "Energy and Maximum Norm Estimates for Nonlinear Conservation Laws," Tech. rep., RIACS Tech. Report 91.01, 1994.

${ }^{24}$ Yee, H. and Sjögreen, B., "Designing Adaptive Low Dissipative High Order Schemes for Long-Time Integrations," In Turbulent Flow Computation, edited by E. D. D. . B. Geurts, Kluwer Academic Publisher, 2002, also RIACS Technical Report TR01-28, Dec. 2001.

${ }^{25}$ Yee, H. and Sjögreen, B., "Development of Low Dissipative High Order Filter Schemes for Multiscale Navier-Stokes/MHD Systems," J. Comput. Phys., Vol. 225, 2007, pp. 910-934.

${ }^{26}$ Sjögreen, B. and Yee, H., "Multiresolution Wavelet Based Adaptive Numerical Dissipation Control for Shock-Turbulence Computation," Riacs technical report tr01.01, NASA Ames research center, Oct 2000, also J. Scient. Comput., 20 (2004) 211-255.

${ }^{27}$ H.C. Yee, N. S. and Djomehri, M., "Low Dissipative High Order Shock-Capturing Methods Using Characteristic-Based Filters," J. Comput. Phys., Vol. 150, 1999, pp. 199-238.

${ }^{28}$ Olsson, P., "Summation by Parts, Projections and Stability, I," Math. Comp., Vol. 64, 1995, pp. 1035-1065.

${ }^{29}$ Chesshire, G. and Henshaw, W., "Composite Overlapping Meshes for the Solution of Partial Differential Equations," J. Comput. Phys., Vol. 90, No. 1, 1990, pp. 1-64.

${ }^{30}$ Park, C., "Review of Chemical-Kinetic Problems of Future NASA Mission, I: Earth Entries," Journal of Thermophysics an Transfer, Vol. 7, July-Sept 1993, pp. 385-398.

${ }^{31}$ Lani, A., Yee, H. C., and Sjögreen, B., "High order simulation of hypersonic nonequilibrium flows on overset grids," to be included in CTR Annual Research Briefs 2010.

${ }^{32}$ Barnhardt, M. and Candler, G., "Detached Eddy Simulation of the reentry-F flight experiment," 46th AIAA Aerospace Sciences Meeting, 7-10 January, Reno (Nevada), AIAA 2008-625, 2008.

${ }^{33}$ MacLean, M., Mundy, E., Wadhams, T., Holden, M., Barnhardt, M., and Candler, G., "Experimental and numerical study of laminar and turbulent base flow on spherical capsule," 47th AIAA Aerospace Sciences Meeting, 5-8 January, Orlando (Florida), AIAA 2009-783, 2009.

${ }^{34}$ Sinha, K., Barnhardt, M., and Candler, G., "Detached Eddy Simulation of hypersonic Base Flows with application to Fire II experiments," 38th AIAA Fluid Dynamics Conference and Exhibit, 28 June - 1 July, Portland (Oregon), AIAA 2004-2633-853, 2004. 